\title{
Association between maternal ritodrine hydrochloride administration during pregnancy and childhood wheezing up to three years of age: The Japan Environment and Children's Study
}

\author{
Tsuyoshi Murata ${ }^{1}$, Hyo Kyozuka ${ }^{1}$, Shun Yasuda ${ }^{2}$, Toma Fukuda ${ }^{2}$, Akiko Yamaguchi ${ }^{2}$ \\ Hajime Maeda $^{1}$, Akiko Sato ${ }^{3}$, Yuka Ogata ${ }^{4}$, Kosei Shinoki ${ }^{4}$, Mitsuaki Hosoya ${ }^{1}$, Seiji \\ Yasumura $^{1}$, Koichi Hashimoto ${ }^{1}$, Hidekazu Nishigori ${ }^{1}$, and Keiya Fujimori ${ }^{1}$ \\ ${ }^{1}$ Fukushima Medical University School of Medicine \\ ${ }^{2}$ Fukushima Medical University \\ ${ }^{3}$ Fukushima Regional Center for the Japan Environmental and Children's Study \\ ${ }^{4}$ Fukushima Regional Center for the Japan Environmental and Children's Study
}

March 17, 2021

\begin{abstract}
Background: The effects of maternal ritodrine hydrochloride administration (MRA) during pregnancy on fetuses and offspring are not entirely clear. The present study aimed to evaluate the association between MRA and childhood wheezing using data from a nationwide Japanese birth cohort study. Methods: This study retrospectively analyzed data from the Japan Environment and Children's Study, a nationwide birth cohort study, conducted between 2011 and 2014. Data of women with singleton births after 22 weeks of gestation were analyzed. The participants were divided according to MRA status. Considering childhood factors affecting the incidence of wheezing, a logistic regression model was used to calculate adjusted odds ratios for "wheezing ever," diagnosis of asthma in the last 12 months, and "asthma ever" in women with MRA, with women who did not receive MRA as the reference. Participants were stratified by term births, and adjusted odds ratios for outcomes were calculated using a logistic regression model. Results: A total of 68,123 participants were analyzed. The adjusted odds ratio for wheezing ever was 1.17 (95\% confidence interval, 1.12-1.22). The adjusted odds ratios for the other outcomes did not significantly increase after adjusting for childhood factors. The same tendency was confirmed after excluding women with preterm births. Conclusion: MRA was associated with an increased incidence of childhood wheezing up to three years, irrespective of term births or preterm births. It is important that perinatal physicians consider both the adverse maternal side effects of MRA and its potential effects on the offspring's childhood.
\end{abstract}

Association between maternal ritodrine hydrochloride administration during pregnancy and childhood wheezing up to three years of age: The Japan Environment and Children's Study

Tsuyoshi Murata ${ }^{1,2}$, Hyo Kyozuka ${ }^{1,2}$, Shun Yasuda ${ }^{1,2}$, Toma Fukuda ${ }^{1,2}$, Akiko Yamaguchi ${ }^{1,2}$, Hajime Maeda $^{1,3}$, Akiko Sato ${ }^{1}$, Yuka Ogata ${ }^{1}$, Kosei Shinoki ${ }^{1}$, Mitsuaki Hosoya ${ }^{1,3}$, Seiji Yasumura ${ }^{1,4}$, Koichi Hashimoto $^{1,3}$, Hidekazu Nishigori ${ }^{1,5}$, Keiya Fujimori ${ }^{1,2}$, and The Japan Environment and Children's Study (JECS) Group

${ }^{1}$ Fukushima Regional Center for the Japan Environment and Children's Study, 1 Hikarigaoka, Fukushima 960-1295, Japan

${ }^{2}$ Department of Obstetrics and Gynecology, Fukushima Medical University School of Medicine, 1 Hikarigaoka, Fukushima 960-1295, Japan 
${ }^{3}$ Department of Pediatrics, Fukushima Medical University School of Medicine, 1 Hikarigaoka, Fukushima 960-1295, Japan

${ }^{4}$ Department of Public Health, Fukushima Medical University School of Medicine, 1 Hikarigaoka, Fukushima 960-1295, Japan

${ }^{5}$ Fukushima Medical Center for Children and Women, Fukushima Medical University, 1 Hikarigaoka, Fukushima 960-1295, Japan

Running title: Ritodrine and childhood wheezing

\section{Correspondence}

Tsuyoshi Murata, MD

Department of Obstetrics and Gynecology, Fukushima Medical University School of Medicine, 1 Hikarigaoka, Fukushima 960-1295, Japan

Tel.: +81-24-547-1290, Fax: +81-24-548-3878

E-mail: tuyoshim@fmu.ac.jp

Manuscript word count: 2,598 words

Abstract word count: 246 words

Number of figures: 1

Number of tables: 3

Number of references: 38

Material in the electronic repository: not applicable

Hosted file

2.Murata main text final.pdf available at https://authorea.com/users/327152/articles/513952association-between-maternal-ritodrine-hydrochloride-administration-during-pregnancyand-childhood-wheezing-up-to-three-years-of-age-the-japan-environment-and-children-sstudy

Hosted file

3.Murata figure final.pdf available at https://authorea.com/users/327152/articles/513952association-between-maternal-ritodrine-hydrochloride-administration-during-pregnancyand-childhood-wheezing-up-to-three-years-of-age-the-japan-environment-and-children-sstudy 